\title{
SCIENTIFIC REPORTS

\section{OPEN Association of multiple glycemic parameters at intensive care unit admission with mortality and clinical outcomes in critically ill patients}

\author{
Priscila Bellaver ${ }^{1,2}$, Ariell F. Schaeffer ${ }^{3}$, Diego P. Dullius ${ }^{4}$, Marina V. Viana $^{5}$, \\ Cristiane B. Leitão ${ }^{1,6}$ \& Tatiana H. Rech $\mathbb{1}^{1,5^{*}}$
}

The aim of the present study was to investigate the association of multiple glycemic parameters at intensive care unit (ICU) admission with outcomes in critically ill patients. Critically ill adults admitted to ICU were included prospectively in the study and followed for 180 days until hospital discharge or death. Patients were assessed for glycemic gap, hypoglycemia, hyperglycemia, glycemic variability, and stress hyperglycemia ratio (SHR). A total of 542 patients were enrolled ( $30 \%$ with preexisting diabetes). Patients with glycemic gap $>80 \mathrm{mg} / \mathrm{dL}$ had increased need for renal replacement therapy (RRT; $37.7 \%$ vs. $23.7 \%, p=0.025)$ and shock incidence $(54.7 \%$ vs. $37.4 \%, p=0.014)$. Hypoglycemia was associated with increased mortality ( $54.8 \%$ vs. $35.8 \%, p=0.004)$, need for RRT ( $45.1 \%$ vs. $22.3 \%$, $\mathrm{p}<0.001$ ), mechanical ventilation ( $\mathrm{MV} ; 72.6 \%$ vs. $57.5 \%, \mathrm{p}=0.024)$, and shock incidence ( $62.9 \%$ vs. $35.8 \%, p<0.001)$. Hyperglycemia increased mortality ( $44.3 \%$ vs. $34.9 \%, p=0.031)$. Glycemic variability $>40 \mathrm{mg} / \mathrm{dL}$ was associated with increased need for RRT ( $28.3 \%$ vs. $14.4 \%, \mathrm{p}=0.002)$ and shock incidence $(41.4 \%$ vs.31.2\%, $p=0.039)$. In this mixed sample of critically ill subjects, including patients with and without preexisting diabetes, glycemic gap, glycemic variability, and SHR were associated with worse outcomes, but not with mortality. Hypoglycemia and hyperglycemia were independently associated with increased mortality.

In critically ill patients, hyperglycemia is an adaptive metabolic response to acute stress ${ }^{1,2}$. Optimal glycemic targets in the ICU setting are controversial and seem to be related to previous patient metabolic status ${ }^{3-9}$. As glycated hemoglobin (HbAlc) is not affected by the onset of acute illness, it can be used to estimate chronic glycemic control in critically ill patients ${ }^{10}$. In the intensive care setting, patients with higher HbAlc have higher mortality 11,12 . However, patients with poor chronic glycemic control have worse outcomes when treated with intensive glycemic control ${ }^{13}$, suggesting that chronic hyperglycemia might be able to generate cellular mechanisms that protect against damage mediated by acute hyperglycemia during critical illness ${ }^{14}$. Therefore, the question is more complex than selecting the "optimal glycemic target", as the interplay between multiple domains of glycemic control might be more relevant. In this sense, glycemic variability, which reflects the magnitude of glycemic excursions during the day, has been associated with unfavorable outcomes in critical illness, including higher mortality ${ }^{15,16}$.

In addition, to separate preexisting hyperglycemia from stress-induced hyperglycemia (elevated blood glucose that reverts to normal after disease and inflammation subsides), the glycemic gap and the stress hyperglycemia ratio (SHR) have been proposed as predictors of adverse outcomes in the ICU. The glycemic gap, defined as the difference between blood glucose at ICU admission and the estimated mean blood glucose derived from $\mathrm{HbAlc}$

${ }^{1}$ Graduate Program in Medical Sciences: Endocrinology, Universidade Federal do Rio Grande do Sul (UFRGS), Porto Alegre, RS, Brazil. 'Department of Internal Medicine, Hospital de Clínicas de Porto Alegre, Porto Alegre, RS, Brazil. ${ }^{3}$ School of Medicine, Universidade Federal do Rio Grande do Sul (UFRGS), Porto Alegre, RS, Brazil. ${ }^{4}$ Department of Surgery, Hospital de Clínicas de Porto Alegre, Porto Alegre, RS, Brazil. 5 Intensive Care Unit, Hospital de Clínicas de Porto Alegre, Porto Alegre, RS, Brazil. 'Endocrine Division, Hospital de Clínicas de Porto Alegre, Porto Alegre, RS, Brazil. *email: threch@hcpa.edu.br 
values, is associated with worse prognosis in specific populations of critically ill patients, such as patients with acute myocardial infarction, community-acquired pneumonia, and hepatic abscess ${ }^{17-19}$. Moreover, a glycemic gap above $80 \mathrm{mg} / \mathrm{dL}$ is associated with higher hospital mortality in critically ill patients with $\mathrm{DM}^{20}$, but its value as a prognostic tool in mixed medical-surgical sample of critically ill subjects is unknown. The SHR is calculated by dividing the blood glucose on admission by the estimated average glucose derived from HbA1c. Studies suggest that an SHR $>1.1$ is a better predictor for worse outcomes in critical illness than the absolute mean blood glucose $^{21}$.

Within this context, the aim of the present study is to investigate the association of multiple glycemic parameters (glycemic gap, hypoglycemia, hyperglycemia, glycemic variability, and SHR) at ICU admission with clinical outcomes in critically ill patients with and without diabetes. The primary endpoint was mortality. Secondary endpoints were need for renal replacement therapy (RRT), incidence of shock, need for mechanical ventilation (MV), time spent on MV, length of stay (LOS) in the hospital, LOS in the ICU, and need for ICU readmission.

\section{Results}

Study population characteristics. A total of 542 consecutive patients admitted to the ICU were included in the study. Their main characteristics are summarized in Supplementary Table S1. Briefly, 52.5\% were male, and the mean age was $59 \pm 15$ years; $42.4 \%$ were admitted to ICU from the emergency department. ICU admissions were for medical reasons in $84.3 \%$ and for surgical reasons in $15.7 \%$, with acute respiratory failure as the leading cause $(23.6 \%)$. The most common primary coexisting condition was hypertension (54\%), followed by DM (30\%). Mean serum glucose at admission was higher in nonsurvivors than in survivors (146 $\pm 73 \mathrm{vs} .132 \pm 60 \mathrm{mg} /$ $\mathrm{dL}, \mathrm{p}=0.023)$, but HbAlc was not $(5.8 \pm 1.7$ vs. $5.5 \pm 1.4 \% \mathrm{p}=0.058)$. The overall mortality rate was $38.2 \%$. When analyzing patients with DM separately, the mortality rate was similar regardless of preexisting DM ( $43 \%$ vs. $38.2 \% ; \mathrm{p}=0.193)$. In patients with $\mathrm{DM}(\mathrm{n}=163 ; \mathrm{n}=4$ type $1 \mathrm{DM} ; \mathrm{n}=159$ type $2 \mathrm{DM})$, mean blood glucose and HbAlc were similar in survivors and nonsurvivors (mean blood glucose: $166 \pm 87$ vs. $187 \pm 96, p=0.16$; HbAlc $6.8 \pm 1.8 \%$ vs. $6.9 \pm 2.4, \mathrm{p}=0.705)$.

Glycemic parameters and mortality. The glycemic gap varied widely, from -159 to 400 , with a median value of $11(-15$ to 42$) \mathrm{mg} / \mathrm{dL}$ in the overall population and no difference between survivors and nonsurvivors was observed (Fig. 1A). When analyzing patients with DM separately, the glycemic gap was 13 ( -37 to 61 ) $\mathrm{mg} /$ $\mathrm{dL}$, and no differences were observed between survivors and nonsurvivors. Similarly, in patients without preexisting DM, the glycemic gap was not different in survivors and nonsurvivors. When stratifying patients by a glycemic gap cutoff of $80 \mathrm{mg} / \mathrm{dL}$, a trend toward higher mortality was observed in subjects with wider glycemic gaps ( $49 \%$ vs. $37 \%$; $p=0.089)$. Data related to glycemic gap cutoff of $>40 \mathrm{mg} / \mathrm{dL}$ and $>60 \mathrm{mg} / \mathrm{dL}$ are summarized in Supplementary Table S2.

More patients who died had hypoglycemia $(54.8 \%$ vs. $35.8 \%, \mathrm{p}=0.004)$ and hyperglycemia $(44.3 \%$ vs. $34.9 \%$, $\mathrm{p}=0.031)$ compared to survivors.

In the entire sample, mean glycemic variability was 67 (41 to 112$) \mathrm{mg} / \mathrm{dL}$, with higher values in nonsurvivors (Fig. 1B). This difference is not demonstrated in patients with preexisting DM, but is clearly seem in patients without DM. When patients with a glycemic variability cutoff of $40 \mathrm{mg} / \mathrm{dL}$ were analyzed in separate, no difference in mortality was observed in subjects with higher glycemic amplitudes ( $34 \%$ vs. $39 \% ; \mathrm{p}=0.228)$. Data related to variability $>60 \mathrm{mg} / \mathrm{dL}$ and $>80 \mathrm{mg} / \mathrm{dL}$ are summarized in Supplementary Table S3. Overall SHR was $1.1( \pm 0.5)$, with no difference between survivors and nonsurvivors (Fig. 1C), independently or preexisting DM or not.

Glycemic parameters and other outcomes. When comparing patients with glycemic gap above and below $80 \mathrm{mg} / \mathrm{dL}$, the group with higher values had increased need for RRT and a higher incidence of shock (Table 1).

The presence of hypoglycemia within the first 24 hours of ICU admission was associated with increased need for RRT, increased need for MV, and higher incidence of shock. Length of hospital stay was shorter in this population. The presence of hyperglycemia on admission was associated with increased need for MV. Glycemic variability during the first 24 hours of admission was associated with an increased need for RRT and a higher incidence of shock. These results are summarized in Table 2 and Supplementary Table S3. SHR $>1.1$ was associated with greater need for MV, but with a shorter time spent on MV and longer ICU stay (Table 1). Interestingly, no glycemic control parameters were associated with the need for ICU readmission (Tables 1 and 2).

Magnitude of the association between glycemic parameters and outcomes. Figure 2 presents the relative risks (RR) for outcomes according to each glycemic parameter. Glycemic gap $>80 \mathrm{mg} / \mathrm{dL}$ was associated with an increased need for RRT and significantly increased the incidence of shock.

Mean blood glucose increased risk of death, but HbAlc did not. Hypoglycemia was the parameter with the highest RR for mortality, followed by hyperglycemia and glycemic variability. Hypoglycemia also yielded the highest RR for the need for RRT. Glycemic variability was also associated with an increased need for RRT. Besides glycemic gap $>80 \mathrm{mg} / \mathrm{dL}$, two other glycemic parameters significantly increased the incidence of shock: hypoglycemia and glycemic variability. Regarding the need for MV, hypoglycemia was associated with the highest risk, followed by hyperglycemia. Glycemic variability also increased the need for MV. No glycemic parameter was associated with risk of ICU readmission, as shown in Supplementary Fig. S1.

Cox regression multivariate analysis with adjustment for SAPS 3 was calculated for the glycemic parameters associated with mortality in the univariate models. Three glycemic parameters were associated with increased mortality in the univariate analysis: hypoglycemia (Table 2), hyperglycemia (Table 2) and glucose variability (cutoff $>60 \mathrm{mg} / \mathrm{dL}$ and $>80 \mathrm{mg} / \mathrm{dL}$, Supplementary Table S3). Hypoglycemia and hyperglycemia remained associated with mortality after adjustments (HR 1.68; 95\%CI 1.16 to $2.44, \mathrm{p}=0.006$ and HR 1.37; 95\%CI 1.04 to 1.81 , 
A
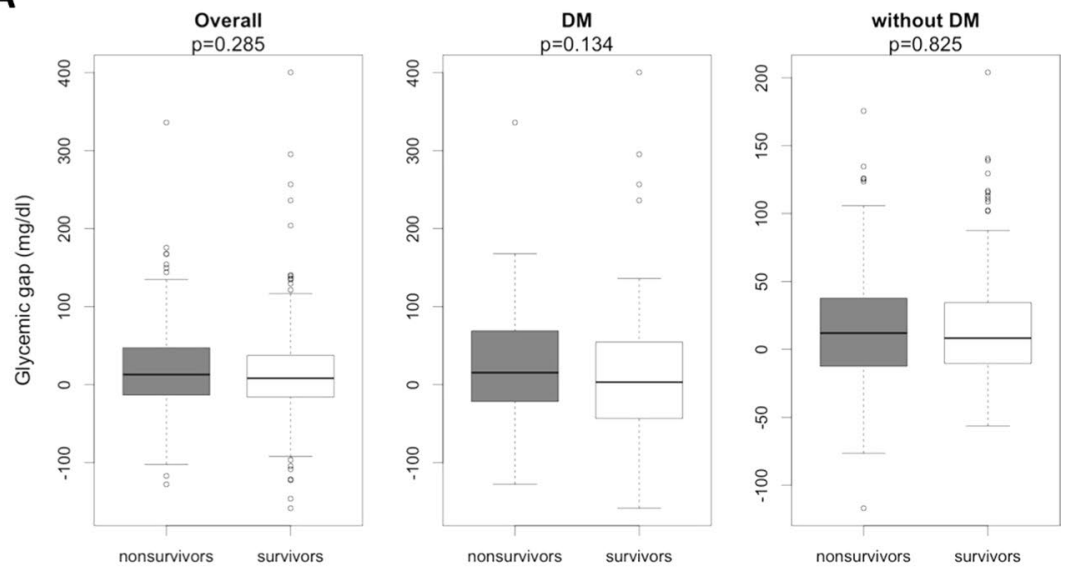

\section{B}
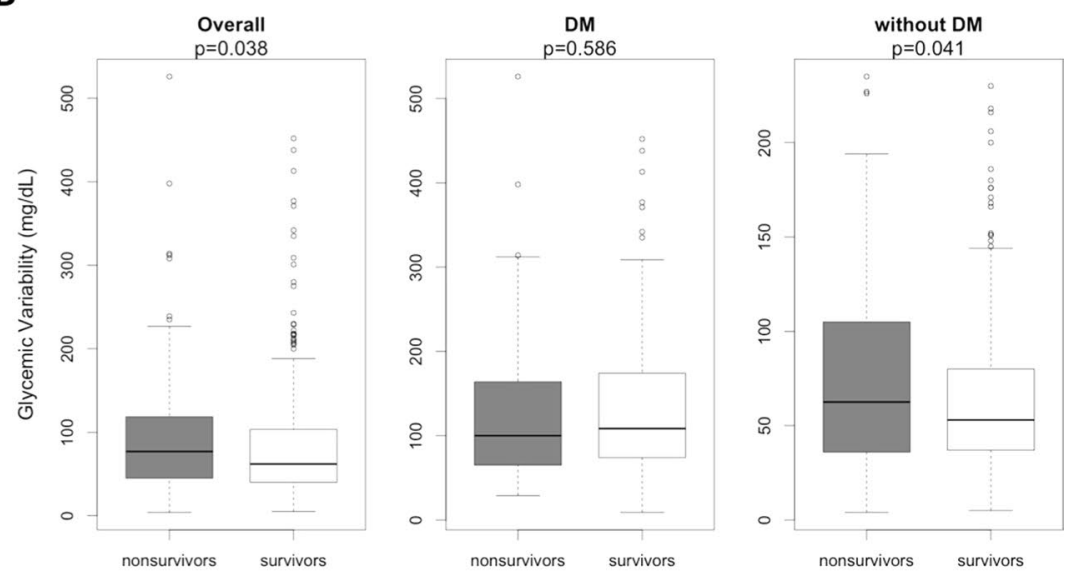

C
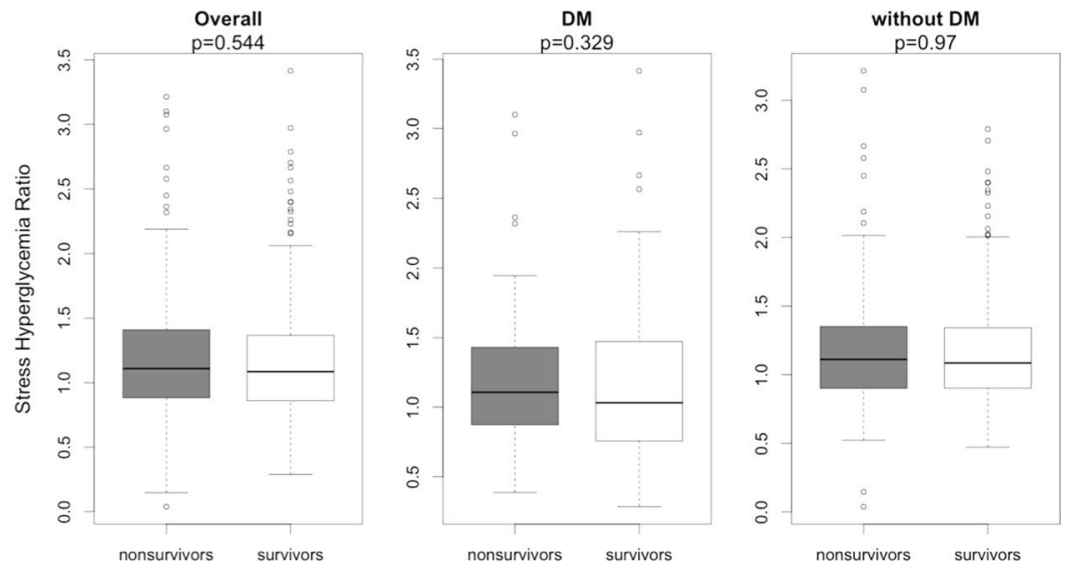

Figure 1. Box plot demonstrating the effect of glycemic gap (A), glycemic variability (B), and stress hyperglycemia ratio $(\mathbf{C})$ on mortality in overall population, in patients with DM (diabetes mellitus), and in patients without DM. Values are median and interquartile range; dots represent outliers. Figures were constructed using R version 3.5.2 (The R Foundation, version 3.5.1, 2018).

$\mathrm{p}=0.026$, for hypoglycemia and hyperglycemia, respectively) (Fig. 3A,B), but glycemic variability did not (HR $1.14 ; 95 \% \mathrm{CI} 0.86$ to $1.51, \mathrm{p}=0.366$ for variability $>60 \mathrm{mg} / \mathrm{dL}$ and $\mathrm{HR} 1.25 ; 95 \% \mathrm{CI} 0.95$ to $1.64, \mathrm{p}=0.104$ for variability $>80 \mathrm{mg} / \mathrm{dL}$ ). 


\begin{tabular}{|c|c|c|c|c|c|c|}
\hline \multirow[b]{2}{*}{ Outcomes } & \multicolumn{2}{|l|}{ Glycemic gap } & \multirow[b]{2}{*}{$\mathbf{p}$} & \multicolumn{2}{|l|}{ SHR } & \multirow[b]{2}{*}{$\mathbf{p}$} \\
\hline & $\begin{array}{l}<80 \mathrm{mg} / \mathrm{dL} \\
(\mathrm{n}=489)\end{array}$ & $\begin{array}{l}>80 \mathrm{mg} / \mathrm{dL} \\
(\mathrm{n}=53)\end{array}$ & & $<1.1(n=267)$ & $\begin{array}{l}>1.1 \\
(n=275)\end{array}$ & \\
\hline Mortality (n, \%) & $181(37)$ & $26(49)$ & 0.087 & $96(36)$ & $111(40)$ & 0.247 \\
\hline $\begin{array}{l}\text { Need for RRT (n, \%) } \\
\text { Shock incidence (n, \%) }\end{array}$ & $\begin{array}{l}116(23.7) \\
183(37.4)\end{array}$ & \begin{tabular}{|l|}
$20(37.7)$ \\
$29(54.7)$
\end{tabular} & $\begin{array}{l}0.025 \\
0.014\end{array}$ & $\begin{array}{l}62(23) \\
96(36)\end{array}$ & \begin{tabular}{|l|}
$74(27)$ \\
$116(42)$
\end{tabular} & $\begin{array}{l}0.287 \\
0.112\end{array}$ \\
\hline Need for MV $(n, \%)$ & $286(58.4)$ & $36(67.9)$ & 0.184 & $141(52.8)$ & $181(65.8)$ & 0.001 \\
\hline Time on MV (days) & $4(1$ to 8$)$ & $4.5(2$ to 8$)$ & 0.117 & $3(1$ to 8$)$ & $2(4$ to 8.5$)$ & $<0.001$ \\
\hline LOS, hospital (days) & 20 (10 to 35$)$ & 20 (10 to 34.7$)$ & 0.585 & $19(10.7$ to 32.5$)$ & 21 (9 to 38$)$ & 0.263 \\
\hline LOS, ICU (days) & 7 (3 to 12$)$ & $7.5(4.25$ to 11$)$ & 0.126 & $6(3$ to 11$)$ & $8(4$ to 12$)$ & 0.005 \\
\hline ICU readmission $(\mathrm{n}, \%)$ & $62(12.6)$ & $6(11.3)$ & 0.770 & $30(11.2)$ & $38(13.8)$ & 0.339 \\
\hline
\end{tabular}

Table 1. Effects of stress-induced hyperglycemia on clinical outcomes. SHR: stress hyperglycemia ratio; RRT: renal replacement therapy; MV: mechanical ventilation; LOS: length of stay; ICU: intensive care unit. Glycemic gap was calculated by the difference between the serum glucose at ICU admission and the estimated mean blood glucose derived from HbAlc. SHR was defined by the ratio between serum glucose at admission and the estimated mean blood glucose derived from HbA1c. Values are mean \pm SD or median and interquartile range.

\begin{tabular}{|c|c|c|c|c|c|c|c|c|c|}
\hline \multirow[b]{3}{*}{ Outcomes } & \multicolumn{2}{|c|}{ Hypoglycemia } & \multirow[b]{3}{*}{$\mathbf{p}$} & \multicolumn{2}{|c|}{ Hyperglycemia } & \multirow[b]{3}{*}{$\mathbf{p}$} & \multicolumn{2}{|c|}{ Glycemic variability } & \multirow[b]{3}{*}{$\mathbf{p}$} \\
\hline & \multirow{2}{*}{\begin{tabular}{|l|} 
No \\
$(n=480)$ \\
\end{tabular}} & \multirow{2}{*}{$\begin{array}{l}\text { Yes } \\
(n=62)\end{array}$} & & \multirow{2}{*}{$\begin{array}{l}\text { No } \\
(n=350)\end{array}$} & \multirow{2}{*}{$\begin{array}{l}\text { Yes } \\
(n=192)\end{array}$} & & \multirow{2}{*}{$\begin{array}{l}<40 \mathrm{mg} / \mathrm{dL} \\
(\mathrm{n}=125)\end{array}$} & \multirow{2}{*}{$\begin{array}{l}>40 \mathrm{mg} / \mathrm{dL} \\
(\mathrm{n}=417)\end{array}$} & \\
\hline & & & & & & & & & \\
\hline Mortality (n, \%) & $172(35.8)$ & $34(54.8)$ & 0.004 & $122(34.9)$ & $85(44.3)$ & 0.031 & $42(33.6)$ & $165(39.5)$ & 0.228 \\
\hline Need for RRT (n, \%) & $107(22.3)$ & $28(45.1)$ & $<0.001$ & $81(23.1)$ & $55(28.6)$ & 0.158 & $18(14.4)$ & $118(28.3)$ & 0.002 \\
\hline Shock incidence $(\mathrm{n}, \%)$ & $172(35.8)$ & $39(62.9)$ & $<0.001$ & $128(36.6)$ & $84(43.7)$ & 0.101 & $39(31.2)$ & $173(41.4)$ & 0.039 \\
\hline Need for MV (n, \%) & $276(57.5)$ & $45(72.6)$ & 0.024 & $195(55.7)$ & $127(66.1)$ & 0.018 & $64(51.2)$ & $258(61.8)$ & 0.033 \\
\hline Time on MV (days) & $4(2$ to 8$)$ & $2(1$ to 6$)$ & 0.365 & $4(1$ to 8$)$ & $4(2$ to 7.7$)$ & 0.014 & $4.5(1.2$ to 9$)$ & $4(1$ to 8$)$ & 0.17 \\
\hline LOS, hospital (days) & $21(11$ to 36$)$ & $14(5$ to 30$)$ & 0.024 & 21 (11 to 37$)$ & $17.5(8$ to 33.5$)$ & 0.498 & $22(12-35)$ & $19(9$ to 36$)$ & 0.575 \\
\hline LOS, ICU (days) & $7(4$ to 12$)$ & $5(2$ to 8.5$)$ & 0.117 & $7(3$ to 12$)$ & $7(4$ to 11$)$ & 0.183 & $8(4$ to 12.7$)$ & $7(3$ to 11$)$ & 0.898 \\
\hline ICU readmission $(\mathrm{n}, \%)$ & $64(13.3)$ & $4(6.5 \%)$ & 0.123 & $41(11.7)$ & $27(14)$ & 0.43 & $14(11.2)$ & $54(12.9)$ & 0.604 \\
\hline
\end{tabular}

Table 2. Effects of hypoglycemia, hyperglycemia, and glycemic variability on clinical outcomes. RRT: renal replacement therapy; MV: mechanical ventilation; LOS: length of stay; ICU: intensive care unit. Hypoglycemia was defined as any serum or capillary glucose $<70 \mathrm{mg} / \mathrm{dL}$ during the first ICU day. Hyperglycemia was defined as any serum glucose $>140 \mathrm{mg} / \mathrm{dL}$ at ICU admission. Glycemic variability was calculated as the absolute difference in capillary blood glucose during the first ICU day. Values are mean \pm SD or median and interquartile range.

\section{Discussion}

In this sample of unselected, prospectively followed critically ill patients, including those with and without preexisting diabetes, multiple glycemic parameters at ICU admission were associated with worse outcomes. Higher glycemic gap, a marker of stress-induced hyperglycemia, increased the need for RRT and incidence of shock, but had not impact on mortality. The presence of hypoglycemia in the first 24 hours of ICU admission was associated with the highest RR for worse outcomes, including increased mortality; a single episode of hypoglycemia doubled the risk of death and increased the incidence of shock and the need for RRT threefold. Additionally, hyperglycemia at ICU admission increased the risk of mortality by approximately $50 \%$. Both hypoglycemia and hyperglycemia were independently associated with mortality.

The association between hyperglycemia and mortality in acutely ill patients, especially in those without diabetes, is well documented in the literature ${ }^{22,23}$. However, it is still not clear if hyperglycemia is a marker of disease severity or a determinant of prognosis by itself. In our study, the fact that hyperglycemia was independently associated with mortality corroborates the later. Furthermore, there is physiological plausibility to justify how high glucose levels contribute to worse outcomes, for instance, the deleterious effects of hyperglycemia on immune system function and the increased proteolysis producing lean tissue breakdown ${ }^{24}$. These two mechanisms might contribute to infectious complications ${ }^{25}$ and ICU-acquired muscular weakness ${ }^{26}$ respectively.

Glycemic gap and SHR are markers of acute-stress hyperglycemia, as they are able to separate the effect of acute, critical illness-associated variations in blood glucose from the previous metabolic state. The present study was the first to analyze the role of glycemic gap and SHR in a general medical-surgical population of ICU patients. Unexpectedly, no increase in mortality was demonstrated in patients with high glycemic gaps or high SHR, even when patients with DM were analyzed separately, which stands in contrast to the findings of previous small studies of glycemic gap in very specific populations critically ill patients with diabetes ${ }^{17-19}$. However, a high glycemic gap doubled the risk of shock and the need for RRT and SHR increased risk of the need for MV and the time spent on MV. The main differences in our study are a larger sample size, the inclusion of a mixed sample of surgical and medical critically ill patients, with and without DM, and with high disease severity scores. Therefore, our sample represents a more heterogeneous population, which increases the clinical applicability of our findings. 


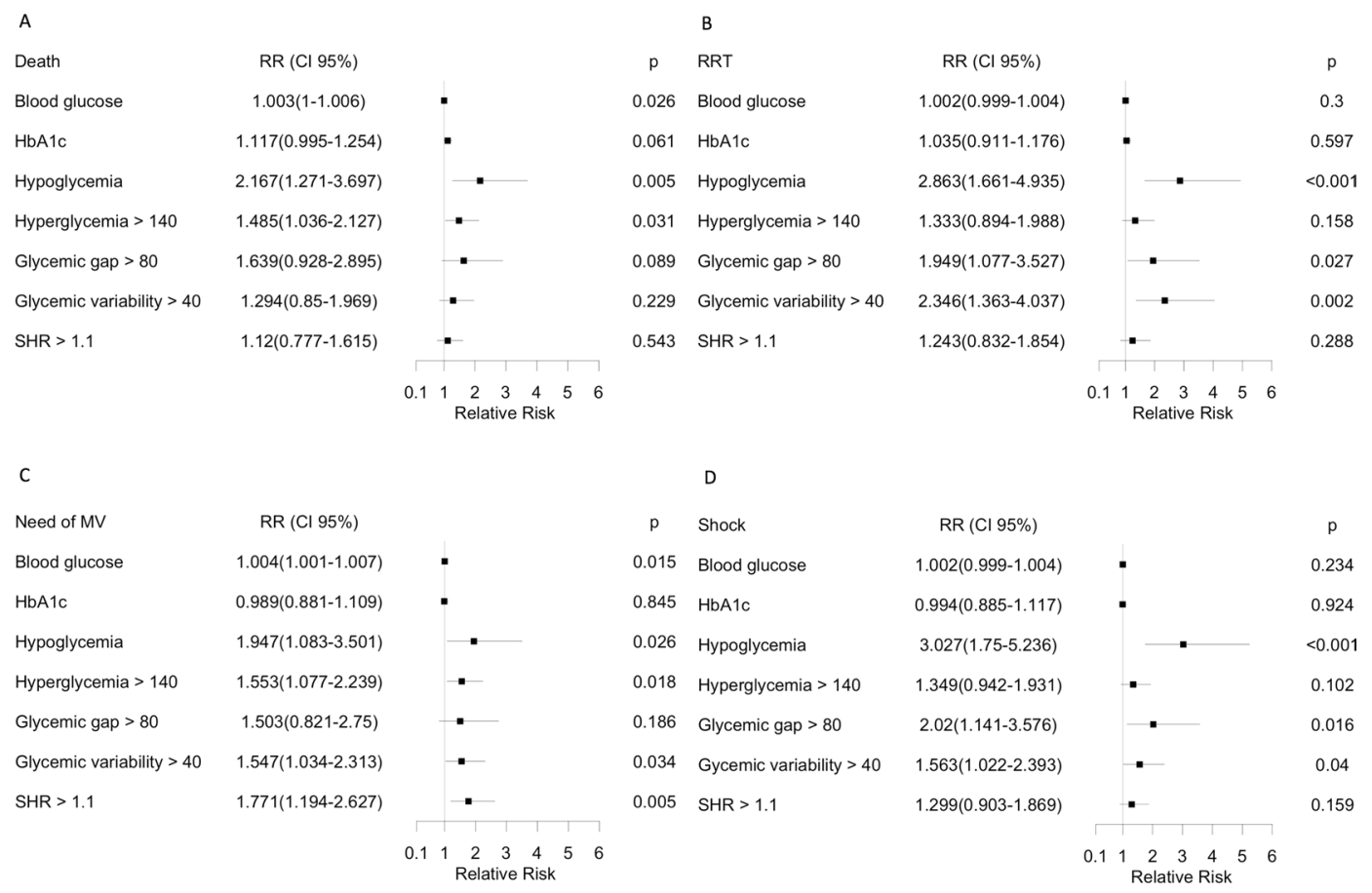

Figure 2. Relative risks for outcomes according to each glycemic parameter. (A) Mortality. (B) Need for renal replacement therapy (RRT). (C) Incidence of shock. (D) Need for mechanical ventilation (MV). HbA1c: glycated hemoglobin. Hypoglycemia was defined as any serum or capillary glucose $<70 \mathrm{mg} / \mathrm{dL}$ during the first ICU day. Hyperglycemia was defined as any serum glucose $>140 \mathrm{mg} / \mathrm{dL}$ at ICU admission. Glycemic gap was calculated by the difference between serum glucose at ICU admission and the estimated mean blood glucose derived from $\mathrm{HbA1c}$. Glycemic variability was calculated as the absolute difference in capillary blood glucose during the first ICU day. SHR (stress of hyperglycemia ratio) was defined by the ratio between serum glucose at admission and the estimated mean blood glucose derived from HbA1c.Values are point estimates with 95\% confidence intervals. Figures were constructed using R version 3.5.2 (The R Foundation, version 3.5.1, 2018).

Some studies suggest that glucose variability during the 24 hours is a better predictor of outcomes in critical illness than mean blood glucose $\mathrm{e}^{15,16,27,28}$. However, our results show that glycemic variability measured in the first 24 hours of admission was associated with worse outcomes, increased need for RRT and MV, and higher incidence of shock, but only hypoglycemia and hyperglycemia were associated with increased mortality after adjustment for disease severity. This contrasting result with the literature might be explained by the metric of glucose variability used in our study (difference of maximum and minimum glucose values). Although, we have to highlight that the best indicator for glucose variability quantification is unsettled ${ }^{29}$ and that a retrospective analysis of the Leuven studies ${ }^{8,9}$ showed that increased daily glucose amplitude was associated with increased mortality ${ }^{30}$. Moreover, glycemic variability was not associated with higher risk of mortality in patients with DM, corroborating the idea that patients exposed to chronic hyperglycemia are adapted to glycemic excursions, possibly developing protective cellular mechanisms against wide blood glucose variations during the course of a critical illness $^{14}$. In our study, the mortality of patients with DM was similar to that of patients without DM, in agreement with the concept of the "diabetic paradox" in the ICU-i.e., the finding that DM is not independently associated with increased risk of mortality in heterogeneous populations of critically ill patients ${ }^{31}$.

This study has limitations. First, glucose monitoring was not continuous, which raises the possibility that some extreme glucose values may have gone unrecorded. However, this is a conservative bias that might have decreased differences in outcomes, further corroborating our findings. Furthermore, glucometers are still largely used because they are practical and low cost. Second, accurate information on diet (including the amount of calories consumed) and on insulin doses administered was not recorded, thus precluding conclusions regarding the influence of carbohydrate intake or insulin therapy on outcomes. However, the association of at least one of the outcomes evaluated herein, hypoglycemia, seems to be independent from insulin use, as suggested by the results of the NICE-SUGAR study ${ }^{32}$. Third, the absence of data on diabetes duration and the low absolute number of patients with type $1 \mathrm{DM}$ prevent any conclusion regarding different underlying pathogenic mechanisms between type 1 and type $2 \mathrm{DM}$ and long term complications over the outcomes.

The main strength of this study is the prospective cohort design, specifically selected to evaluate multiple glycemic parameters simultaneously in a large unselected population of critically ill patients and their associations with clinical outcomes and mortality. Our findings reinforce that, besides mean blood glucose seems to be the most important predictor of outcomes, several other domains of glycemic control are relevant in critical illness. This might be especially important for research in glycemic control, as future trials should evaluate not only glycemic targets, but rather multiple glycemic parameters at the same time. 


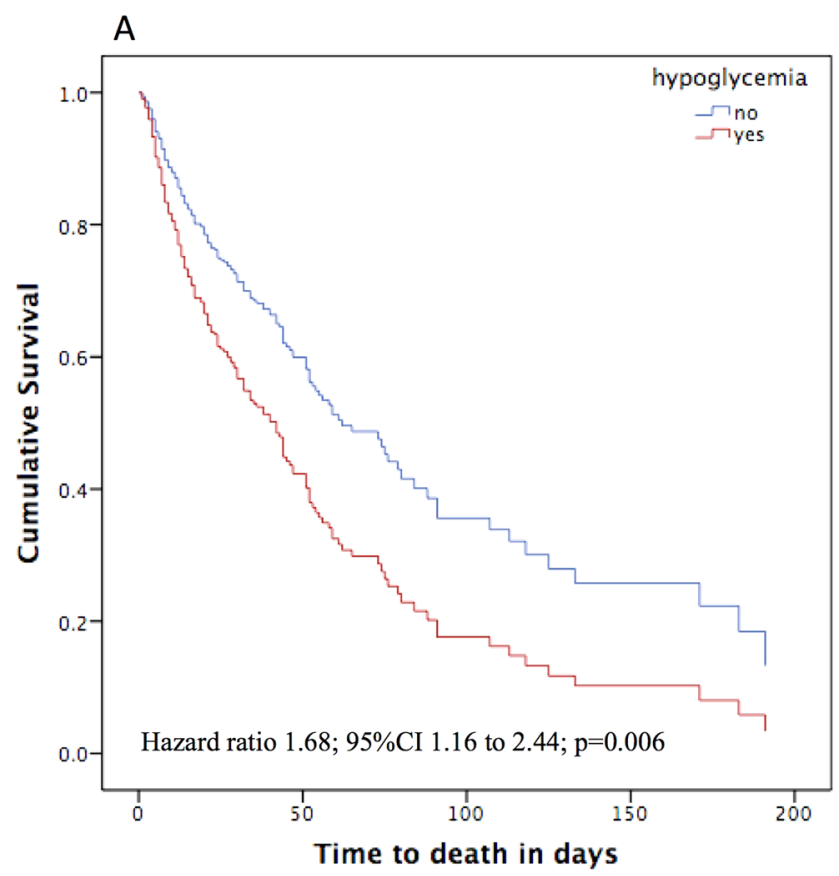

B

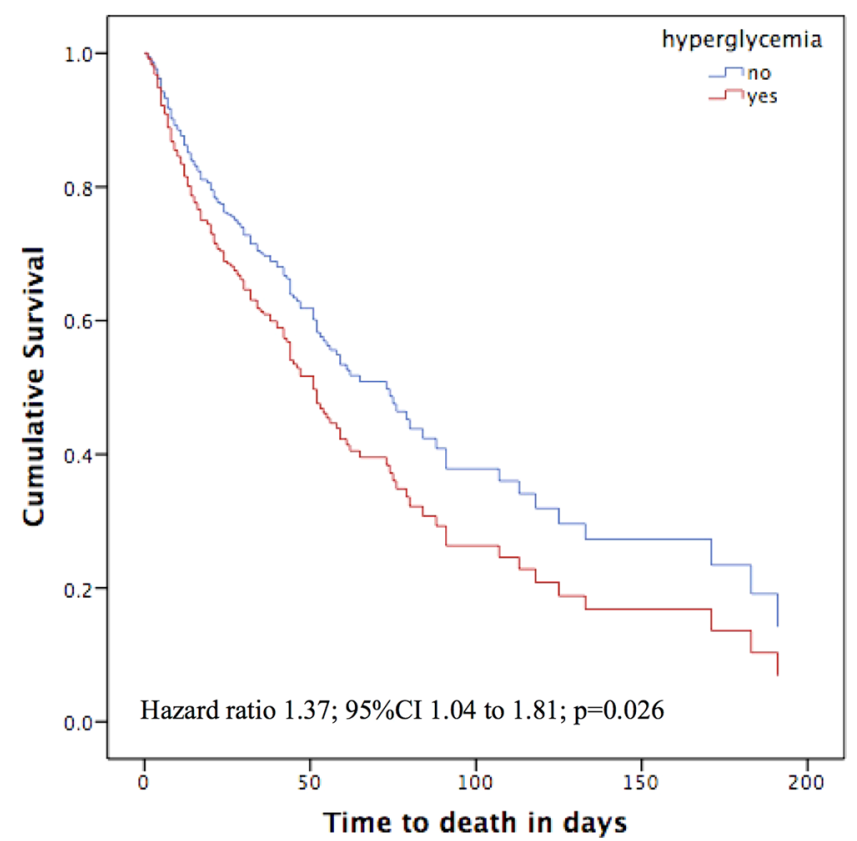

Figure 3. Cumulative survival at 180 days stratified by presence of abnormal blood glucose at ICU admission. (A) Hypoglycemia (defined as any serum or capillary glucose measurement $<70 \mathrm{mg} / \mathrm{dL}$ during the first ICU day). (B) Hyperglycemia (defined as any serum glucose measurement $>140 \mathrm{mg} / \mathrm{dL}$ at ICU admission). Hazard ratios are adjusted for SAPS 3 (Simplified Acute Physiology III) score. Figures were constructed using SPSS 20.0 (Chicago, IL, USA).

In summary, in this mixed medical-surgical sample of critically ill subjects, including patients with and without previous diagnosis of diabetes, glycemic gap was associated with worse clinical outcomes, but had no impact on mortality. Hypoglycemia and hyperglycemia were independently associated with increased mortality and influenced other outcomes, such as incidence of shock and need for RRT and MV. Similarly to glycemic gap, glycemic variability and SHR also negatively affected outcomes, with no impact on mortality. It is common sense that optimal glycemic control should be pursued to reduce the risk of unfavorable outcomes. Then, further research is needed to personalize glycemic control targets in critically ill patients, focusing on a broader view of glucose dysregulation based in multiple parameters rather than in a single, in an effort to reduce the risks of iatrogenic adverse events. 


\begin{abstract}
Methods
Study population. This is a prospective cohort study. The study protocol was approved by the ethics committee at Hospital de Clínicas de Porto Alegre (project number 17-0386). Informed consent was obtained from patients or their legal representatives. From September 2017 to February 2018, critically ill adults (age $>18$ years) admitted to the ICU were prospectively included in the study. The exclusion criteria were pregnancy, diabetic ketoacidosis, hyperosmolar hyperglycemic state, sickle cell anemia, and other hemoglobinopathies. Blood samples were collected at study entry from all patients for random serum blood glucose and HbAlc quantifications, and clinical and laboratory data were recorded for all patients. Simplified Acute Physiology Score 3 (SAPS 3), ranging theoretically from a minimum of 0 points to a maximum of 271 points, with higher scores denoting higher severity ${ }^{33}$, was used to score disease severity.

Hyperglycemia was defined according to the American Diabetes Association (ADA) proposed threshold for in-hospital hyperglycemia as any blood glucose measurement $>140 \mathrm{mg} / \mathrm{dL}^{34-36}$ at ICU admission. Moderate hypoglycemia was any blood or capillary glucose $<70 \mathrm{mg} / \mathrm{dL}$ (ADA definition and same level from the NICE-SUGAR study) $)^{32,34}$ and serious hypoglycemia was $<54 \mathrm{mg} / \mathrm{dL}$ (ADA definition) ${ }^{34}$ during the first day in the ICU. Glucose variability was calculated as the absolute difference in capillary blood glucose during the first day in the ICU ${ }^{15,16}$. In order to separate the effects of a chronically altered metabolic state from those of acute stress hyperglycemia, the glycemic gap and SHR were evaluated. The glycemic gap was calculated by the difference between the ICU admission serum blood glucose and the estimated mean blood glucose (serum blood glucose on ICU admission - estimated mean blood glucose $)^{17,18}$. HbAlc values were used to calculate the estimated mean blood glucose, using the following formula: $(28.7 \times \mathrm{HbA1c})-46.7 \mathrm{mg} / \mathrm{dL}$ (30). The cutoff value of $80 \mathrm{mg} / \mathrm{dL}$ for glycemic gap was based on Liau et al. ${ }^{20}$. SHR was defined by the ratio between serum blood glucose at admission and the estimated mean blood glucose and the cutoff value of 1.1 based on Roberts et al. ${ }^{21}$. Diabetes was defined on the basis of previous diagnosis or when HbAlc was $\geq 6.5 \%{ }^{34}$. HbAlc quantifications were measured for all patients at ICU admission. Previous diagnosis of diabetes was assessed by two researchers (P.B. and A.S) by electronic chart review (hospital and ICU admission notes, previous use of antihyperglycemic agents or insulin, and outpatient visits if needed).

The outcomes of interest were adjudicated by two unblinded researchers (P.B. and A.S) and included the following: mortality (primary endpoint) and need for RRT, incidence of shock, need for MV, time spent on MV, LOS in the hospital, LOS in the ICU, and need for ICU readmission (secondary endpoints). All patients were followed up for 180 days for survival analysis.
\end{abstract}

Biochemical measurements. Blood samples for glucose measurement were collected in tubes with sodium fluoride, centrifuged for $10 \mathrm{~min}$ at $3670 \mathrm{rpm}$, and analyzed by the hexokinase method in a Roche COBAS c702 system (Roche Diagnostics, Mannheim, GE). For HbAlc measurement, blood samples were collected in EDTA tubes, homogenized, and analyzed in BioRAD Variant Turbo II (BioRAD, Hercules, California, USA), processed by HPLC. Glucose values were expressed as $\mathrm{mg} / \mathrm{dL}$, and $\mathrm{HbAlc}$ values as percentage.

Statistical analysis. Categorical variables were expressed as percentages. Data were expressed as mean and standard deviation (SD) if normally distributed, or as median and interquartile range otherwise. Groups were compared using Student's $t$-test, the Mann-Whitney $U$ test, or the chi-square test as appropriate. To assess relative risks of variables of interest and outcomes, univariate linear regression or logistic regression models were constructed depending on the characteristics of the outcomes of interest. A multivariate Cox regression analysis with mortality as outcome was used to calculate hazard ratios (HR), with variables adjusted for disease severity using SAPS 3 score. A sample size of 494 patients was calculated considering a power of $95 \%$ and an $\alpha$-error rate of $5 \%$ to detect a difference in glycemic gap of $42 \mathrm{mg} / \mathrm{dL}$ between survivors and nonsurvivors. There was no missing data. Values were considered statistically significant if $\mathrm{p}<0.05$. Statistical analyses were conducted in SPSS 20.0 (Chicago, IL, USA) and R version 3.5.2 (The R Foundation, version 3.5.1, 2018).

Ethical approval. All experiments were performed in accordance with relevant guidelines and regulations. The study protocol was approved by the Ethics Committee at Hospital de Clínicas de Porto Alegre (project number 17-0386).

Received: 18 August 2019; Accepted: 15 November 2019;

Published online: 06 December 2019

\title{
References
}

1. Van den Berghe, G. Beyond diabetes: saving lives with insulin in the ICU. Int J Obes Relat Metab Disord 26(Suppl 3), S3-8, https:// doi.org/10.1038/sj.ijo.0802171 (2002).

2. Chao, H. Y. et al. Association of In-Hospital Mortality and Dysglycemia in Septic Patients. PLoS One 12, e0170408, https://doi. org/10.1371/journal.pone.0170408 (2017)

3. Preiser, J. C. et al. A prospective randomised multi-centre controlled trial on tight glucose control by intensive insulin therapy in adult intensive care units: the Glucontrol study. Intensive Care Med 35, 1738-1748, https://doi.org/10.1007/s00134-009-1585-2 (2009).

4. Niven, D. J., Rubenfeld, G. D., Kramer, A. A. \& Stelfox, H. T. Effect of published scientific evidence on glycemic control in adult intensive care units. JAMA Intern Med 175, 801-809, https://doi.org/10.1001/jamainternmed.2015.0157 (2015).

5. Wiener, R. S., Wiener, D. C. \& Larson, R. J. Benefits and risks of tight glucose control in critically ill adults: a meta-analysis. JAMA 300, 933-944, https://doi.org/10.1001/jama.300.8.933 (2008).

6. Griesdale, D. E. et al. Intensive insulin therapy and mortality among critically ill patients: a meta-analysis including NICE-SUGAR study data. CMAJ 180, 821-827, https://doi.org/10.1503/cmaj.090206 (2009).

7. Investigators, N.-S. S. et al. Intensive versus conventional glucose control in critically ill patients. N Engl J Med 360, 1283-1297, https://doi.org/10.1056/NEJMoa0810625 (2009). 
8. Van den Berghe, G. et al. Intensive insulin therapy in the medical ICU. N Engl J Med 354, 449-461, https://doi.org/10.1056/ NEJMoa052521 (2006).

9. van den Berghe, G. et al. Intensive insulin therapy in critically ill patients. N Engl J Med 345, 1359-1367, https://doi.org/10.1056/ NEJMoa011300 (2001).

10. Luethi, N. et al. Glycated Hemoglobin Alc Levels Are Not Affected by Critical Illness. Crit Care Med 44, 1692-1694, https://doi. org/10.1097/CCM.0000000000001656 (2016).

11. Kompoti, M. et al. Glycated hemoglobin at admission in the intensive care unit: clinical implications and prognostic relevance. J Crit Care 30, 150-155, https://doi.org/10.1016/j.jcrc.2014.08.014 (2015).

12. Viana, M. V. et al. Contrasting effects of preexisting hyperglycemia and higher body size on hospital mortality in critically ill patients: a prospective cohort study. BMC Endocr Disord 14, 50, https://doi.org/10.1186/1472-6823-14-50 (2014).

13. Egi, M. et al. The interaction of chronic and acute glycemia with mortality in critically ill patients with diabetes. Crit Care Med 39, 105-111, https://doi.org/10.1097/CCM.0b013e3181feb5ea (2011).

14. Klip, A., Tsakiridis, T., Marette, A. \& Ortiz, P. A. Regulation of expression of glucose transporters by glucose: a review of studies in vivo and in cell cultures. FASEB J 8, 43-53, https://doi.org/10.1096/fasebj.8.1.8299889 (1994).

15. Egi, M., Bellomo, R. \& Reade, M. C. Is reducing variability of blood glucose the real but hidden target of intensive insulin therapy? Crit Care 13, 302, https://doi.org/10.1186/cc7755 (2009)

16. Egi, M., Bellomo, R., Stachowski, E., French, C. J. \& Hart, G. Variability of blood glucose concentration and short-term mortality in critically ill patients. Anesthesiology 105, 244-252, https://doi.org/10.1097/00000542-200608000-00006 (2006).

17. Liao, W. I. et al. An Elevated Glycemic Gap is Associated with Adverse Outcomes in Diabetic Patients with Acute Myocardial Infarction. Sci Rep 6, 27770, https://doi.org/10.1038/srep27770 (2016).

18. Liao, W. I. et al. An elevated gap between admission and A1C-derived average glucose levels is associated with adverse outcomes in diabetic patients with pyogenic liver abscess. PLoS One 8, e64476, https://doi.org/10.1371/journal.pone.0064476 (2013).

19. Chen, P. C. et al. An Elevated Glycemic Gap is Associated With Adverse Outcomes in Diabetic Patients With Community-Acquired Pneumonia. Medicine (Baltimore) 94, e1456, https://doi.org/10.1097/MD.0000000000001456 (2015).

20. Liao, W. I. et al. Usefulness of Glycemic Gap to Predict ICU Mortality in Critically Ill Patients With Diabetes. Medicine (Baltimore) 94, e1525, https://doi.org/10.1097/MD.0000000000001525 (2015).

21. Roberts, G. W. et al. Relative Hyperglycemia, a Marker of Critical Illness: Introducing the Stress Hyperglycemia Ratio. J Clin Endocrinol Metab 100, 4490-4497, https://doi.org/10.1210/jc.2015-2660 (2015).

22. Lepper, P. M. et al. Serum glucose levels for predicting death in patients admitted to hospital for community acquired pneumonia: prospective cohort study. BMJ 344, e3397, https://doi.org/10.1136/bmj.e3397 (2012).

23. van Vught, L. A. et al. Admission Hyperglycemia in Critically Ill Sepsis Patients: Association With Outcome and Host Response. Crit Care Med 44, 1338-1346, https://doi.org/10.1097/CCM.0000000000001650 (2016).

24. McCowen, K. C., Malhotra, A. \& Bistrian, B. R. Stress-induced hyperglycemia. Crit Care Clin 17, 107-124, https://doi.org/10.1016/ s0749-0704(05)70154-8(2001).

25. Mizock, B. A. Alterations in fuel metabolism in critical illness: hyperglycaemia. Best Pract Res Clin Endocrinol Metab 15, 533-551, https://doi.org/10.1053/beem.2001.0168 (2001).

26. Zhou, C. et al. Critical illness polyneuropathy and myopathy: a systematic review. Neural Regen Res 9, 101-110, https://doi. org/10.4103/1673-5374.125337 (2014).

27. Egi, M. \& Bellomo, R. Reducing glycemic variability in intensive care unit patients: a new therapeutic target? J Diabetes Sci Technol 3, 1302-1308, https://doi.org/10.1177/193229680900300610 (2009).

28. Zuo, Y. Y., Kang, Y., Yin, W. H., Wang, B. \& Chen, Y. The association of mean glucose level and glucose variability with intensive care unit mortality in patients with severe acute pancreatitis. J Crit Care 27, 146-152, https://doi.org/10.1016/j.jcrc.2011.12.004 (2012).

29. Eslami, S., Taherzadeh, Z., Schultz, M. J. \& Abu-Hanna, A. Glucose variability measures and their effect on mortality: a systematic review. Intensive Care Med 37, 583-593, https://doi.org/10.1007/s00134-010-2129-5 (2011).

30. Meyfroidt, G. et al. Dynamic characteristics of blood glucose time series during the course of critical illness: effects of intensive insulin therapy and relative association with mortality. Crit Care Med 38, 1021-1029, https://doi.org/10.1097/ CCM.0b013e3181cf710e (2010).

31. Krinsley, J. S. \& Fisher, M. The diabetes paradox: diabetes is not independently associated with mortality in critically ill patients. Hosp Pract (1995) 40, 31-35, https://doi.org/10.3810/hp.2012.04.967 (2012).

32. Investigators, N.-S. S. et al. Hypoglycemia and risk of death in critically ill patients. $N$ Engl J Med 367, 1108-1118, https://doi. org/10.1056/NEJMoa1204942 (2012).

33. Moreno, R. P. et al. SAPS 3-From evaluation of the patient to evaluation of the intensive care unit. Part 2: Development of a prognostic model for hospital mortality at ICU admission. Intensive Care Med 31, 1345-1355, https://doi.org/10.1007/s00134-0052763-5 (2005).

34. American Diabetes, A. 15. Diabetes Care in the Hospital: Standards of Medical Care in Diabetes-2019. Diabetes Care 42, S173-S181, https://doi.org/10.2337/dc19-S015 (2019).

35. Kavanagh, B. P. \& McCowen, K. C. Clinical practice. Glycemic control in the ICU. N Engl J Med 363, 2540-2546, https://doi. org/10.1056/NEJMcp1001115 (2010).

36. Smith, F. G., Sheehy, A. M., Vincent, J. L. \& Coursin, D. B. Critical illness-induced dysglycaemia: diabetes and beyond. Crit Care 14, 327, https://doi.org/10.1186/cc9266 (2010).

\section{Acknowledgements}

We thank Édison Moraes Rodrigues for his assistance with SAPS 3 data acquisition. This work was supported by Fundo de Incentivo à Pesquisa e Ensino (FIPE), Hospital de Clínicas de Porto Alegre (project number 17-0386), and Conselho Nacional de Desenvolvimento Científico e Tecnológico (CNPq - process number 420323/2018-0). CBL has received scholarships from Conselho Nacional de Desenvolvimento Científico e Tecnológico (CNPq; PQ-1D).

\section{Author contributions}

P.B. participated in the study conception and design, data acquisition, analysis and interpretation of data, statistical analysis, drafting and revision of the manuscript. A.F.S. participated in data acquisition and analysis. D.P.D. participated in data acquisition. M.V.V. participated in the study design, statistical analysis and revision of the manuscript. C.B.L. participated in the study conception and design, interpretation of data, statistical analysis and revision of the manuscript. T.H.R. participated in the study conception and design, data acquisition, interpretation of data and revision of the manuscript. T.H.R. is the guarantor of this work and, as such, had full access to all data and takes responsibility for the integrity of the data and the accuracy of data analysis. 


\section{Competing interests}

The authors declare no competing interests.

\section{Additional information}

Supplementary information is available for this paper at https://doi.org/10.1038/s41598-019-55080-3.

Correspondence and requests for materials should be addressed to T.H.R.

Reprints and permissions information is available at www.nature.com/reprints.

Publisher's note Springer Nature remains neutral with regard to jurisdictional claims in published maps and institutional affiliations.

(c) (i) Open Access This article is licensed under a Creative Commons Attribution 4.0 International License, which permits use, sharing, adaptation, distribution and reproduction in any medium or format, as long as you give appropriate credit to the original author(s) and the source, provide a link to the Creative Commons license, and indicate if changes were made. The images or other third party material in this article are included in the article's Creative Commons license, unless indicated otherwise in a credit line to the material. If material is not included in the article's Creative Commons license and your intended use is not permitted by statutory regulation or exceeds the permitted use, you will need to obtain permission directly from the copyright holder. To view a copy of this license, visit http://creativecommons.org/licenses/by/4.0/.

(C) The Author(s) 2019 